\title{
Pattern Synthesis of Time-Modulated Sparse Array by an OPM-CVX Algorithm
}

\author{
Lei Liang (iD, Yachao Jiang (iD, Jialing Liu, Hailin Li $(\mathbb{D}$, and Jianjiang Zhou \\ Key Laboratory of Radar Imaging and Microwave Photonics, College of Electronic and Information Engineering, \\ Nanjing University of Aeronautics and Astronautics, Nanjing 210016, China \\ Correspondence should be addressed to Lei Liang; yushan_ll@163.com
}

Received 7 January 2020; Revised 21 February 2020; Accepted 13 March 2020; Published 14 April 2020

Guest Editor: Junpeng Shi

Copyright (c) 2020 Lei Liang et al. This is an open access article distributed under the Creative Commons Attribution License, which permits unrestricted use, distribution, and reproduction in any medium, provided the original work is properly cited.

This paper addresses the constrained multiobjective optimization problem of time-modulated sparse arrays. The synthesis objective is to find an optimal element arrangement and associated excitation strategy of sparse arrays, which realize the balance of radiation power and sideband suppression performance with minimum number of elements, and suppress side lobe level simultaneously. A novel hybrid algorithm based on orthogonal perturbation method and convex optimization (OPM-CVX) for the synthesis of time-modulated sparse antenna array is presented in this paper. In order to satisfy the main lobe beamforming and side lobe suppression of sparse arrays, the proposed method optimizes element positions with minimum array numbers by orthogonal perturbation method and optimizes excitations of array element with dynamic range ratio constraint by convex optimization. Furthermore, a trapezoidal pulse time-modulated switching function is proposed to find the balance of radiation power and sideband suppression performance. The numerical results indicate that the proposed algorithm can be an effective approach for synthesis problems of time-modulated sparse arrays.

\section{Introduction}

The antenna arrays have diverse structures and flexible beam control. It is widely used in radar early warning, meteorology and hydrology, and wireless communication systems [1-3]. Compared with single antenna, antenna arrays make it easier to implement pencil beams, flat top beams, multibeams, and low side lobe patterns. The pencil beam can overcome multipath interference and cochannel interference. Lei [4] optimized the array element positions through an iterative convex optimization algorithm and realized pencil beam and low side lobe in linear and planar arrays. The shaped beam is mainly used to meet the requirements of the radiation area and the intensity of the signal in the specified direction. For example, the flat top beam can ensure the uniformity of the power density at the receiving set, which is significant for improving the reception efficiency and simplifying the design of the rectifier circuit. Cao [5] and Qi [6] synthesized flat top beam with linear array and rectangular planar array, respectively. They effectively controlled the main beam ripple and obtained low side lobe level. In addition, Qi also synthesized a planar array of $10000(100 \times 100)$ elements with half wavelength spacing and verified the applicability of synthesizing shaped beam of large array. However, the cost of the antenna system is increased by the half-wavelength equidistant arrangement.

The sparse array is an important branch in the field of antenna arrays. Compared with uniform antenna arrays, the spacing of array element in sparse array is usually not equal and greater than half wavelength. Under the same array aperture, the sparsely distributed array can obtain better radiation performance with fewer array elements. Morabito [7] synthesized flat top and cosecant patterns using rectangular array with the least number of array elements. Compared with the array with uniform spacing of half wavelength, 58\% of the array elements were saved. Shen [8] and Yan [9] both constrained the aperture of sparse array and synthesized flat top beam and pencil beam. Shen [8] used the unitary matrix pencil method to solve the excitation and element position of linear array, which improves the 
matching accuracy of shaped beam and reduces the computational complexity. However, the dynamic range ratio (DRR) was still greater than 10. Yan [9] adopted the perturbed compressive sampling (PCS) algorithm to optimize the excitation and element position at the same time, which improves the matching accuracy of pattern and obtains a large DRR too. A lot of achievements have been made in the pattern synthesis of sparse arrays. However, the large DRR will increase the implementation difficulty and hardware cost of the feeding network.

The time modulation technology controls the working state of the array element through the RF switch to obtain the equivalent excitation coefficient so that the array element can also obtain good performance of pattern synthesis under the uniform excitation. Poli [10] synthesized a pencil beam pattern and suppressed sideband radiation by optimizing the equivalent excitation of the time-modulated linear array. Alberto Reyna [11] synthesized rectangular and circular sparse arrays with the same aperture size and obtained flat top beam pattern and better feeding network efficiency, but the sideband level was high. Patra [12] synthesized a flat top beam pattern in time-modulated sparse linear array. A combination of static excitation, unequal element spacing, and time is used to obtain a side lobe level of $-40.49 \mathrm{~dB}$ and a sideband level of $-26.46 \mathrm{~dB}$. Through the abovementioned analysis, in addition to obtaining the target pattern, the timemodulated array needs to suppress the sideband radiation caused by the RF switching action. Many scholars have carried out in-depth research on the optimization algorithms of time-modulated arrays and sparse arrays.

The pattern synthesis algorithms of sparse array mainly include evolutionary algorithm, numerical algorithm, and convex optimization algorithm. There are many widely used evolutionary algorithms. D'Urso [13] proposed simulated annealing algorithm to optimize the switch-on duration time, which realized the low side lobe of sparse array with equal spacing and effectively suppressed the sideband radiation. With the constraint of exactly matching the desired pattern of main band, Poli [14] applied the pulse shifting technique to time-modulated arrays and used particle swarm optimization algorithm to suppress the side lobe level and sideband level effectively. However, with the increase of the optimization dimension, the computational complexity of the evolutionary algorithm increases exponentially, the optimization efficiency decreases accordingly, and it is easy to fall into a local optimal solution.

The deterministic numerical methods are widely used with stable optimization solutions. Gassab [15] proposed an efficient mathematical method for pattern synthesis of timemodulated linear array and obtained lower side lobe and sideband than the antenna array optimized by differential evolution algorithm. However, only the switch-on duration time of array element was optimized, while the position of an array element was fixed. Yepes [16] proposed a new hybrid technique to synthesize different shaped beams (narrow beam, flat top beam, and square cosecant beam) for linear array. This technique uses a deterministic algorithm to optimize the nonuniform amplitude excitation and antenna element position and then uses an iterative optimization strategy to calculate the phase of the excitation. Although the deterministic iterative algorithm has high operational stability, its optimization effect largely depends on the initial state of the array. Therefore, joint optimization of parameters such as the position and excitation of an array element can usually achieve better optimization result.

Convex optimization algorithm is applied to pattern synthesis with the advantages of its efficient optimization process and the global optimal result. Echeveste [17] proposed a flat top beam synthesis methodology for planar array by transforming a nonconvex synthesis problem to a convex optimization scheme with conjugate symmetric excitation weights. Under the constraint of the minimum spacing of array elements, Yan [18] proposed a hybrid algorithm of PCS and convex optimization to synthesis a flat top beam of planar array. This method solved the problems of the small element spacing using PCS algorithm and the long or even invalid solving time for the large-scale array using convex optimization algorithm. The above studies all involved transforming a nonconvex problem to a convex problem. This is the key to the application of convex optimization algorithm to pattern synthesis.

Combined with the abovementioned analysis, this paper uses the hybrid algorithm of OPM-CVX to minimize the number of array elements and suppress side lobe and sideband. This algorithm achieves joint optimization of array element excitation and position under the constraint of desired pattern. However, multivariate optimization is a multiobjective multiconstrained nonlinear optimization problem [19]. Taking this issue into consideration, the optimization model is disassembled into linear optimization problems about the number of elements, shaped beam, side lobe, and the balance between sideband and power. The iterative strategy is adopted to achieve the above objectives step by step.

This paper consists of five sections. Some key studies in the field of time-modulated sparse arrays are reviewed in Section 1 and the problem to be addressed by the present study is pointed out. In Section 2, the formula deduction of Fourier coefficient of trapezoidal pulse switching function and the model of optimization are provided. Section 3 gives a step by step solution to the optimization model and describes the optimization process. In Section 4, the effectiveness of this hybrid algorithm is shown by detailed simulation results. Finally, Section 5 presents the conclusions.

\section{Shaped Beam Pattern Synthesis of Time- Modulated Sparse Antenna Array}

2.1. Time-Modulated Sparse Antenna Array. For planar antenna arrays with $N$ identical array elements, as shown in Figure 1, the electric field intensity of far field [20] is given by

$$
E(\theta, \varphi)=\sum_{n=1}^{N} I_{n} e^{j k\left(x_{n} u+y_{n} v\right)} e^{j 2 \pi f_{0} t}
$$

where $I_{n}$ is the excitation coefficient of the $n$ thelement located at $\left(x_{n}, y_{n}\right)$ and $\theta_{n}$ and $\varphi_{n}$ are pitch and azimuth angle, 
respectively. The radiation direction of the array is $(\theta, \varphi)$, $u=\sin \theta \cos \varphi$, and $v=\sin \theta \sin \varphi \cdot k=(2 \pi / \lambda)=\left(2 \pi f_{0} / c\right)$ is the propagation coefficient of the free space with the wavelength $\lambda$, where $f_{0}$ is the carrier frequency.

In time-modulated antenna arrays [21], the $n$th array element is only controlled by $U_{n}(t)$, which is the function of the high speed RF switches. The electric field intensity is redefined as

$$
E(\theta, \varphi)=\sum_{n=1}^{N} I_{n} U_{n}(t) e^{j 2 \pi f_{0} t} e^{j k\left(x_{n} u+y_{n} v\right)} .
$$

The expansion form of time-modulated switching function $U_{n}(t)$ in frequency domain can be expressed as

$$
U_{n}(t)=\sum_{m=-\infty}^{+\infty} a_{m n} e^{j 2 \pi m f_{n} t}, \quad m=0, \pm 1, \pm 2, \ldots,
$$

where $f_{n}=\left(1 / T_{n}\right), T_{n}$ are the time-modulated fundamental frequency and period of the array elements separately and $a_{m n}$ is the Fourier coefficient of the $m$ th sideband. Equation (2) can be rewritten as

$$
E(\theta, \varphi)=\sum_{n=1}^{N} \sum_{m=-\infty}^{+\infty} I_{n} a_{m n} e^{j 2 \pi\left(f_{0}+m f_{n}\right) t} e^{j k\left(x_{n} u+y_{n} v\right)},
$$

where the electric field intensity of the antenna array at the $m$ th sideband is

$$
E_{m}(\theta, \varphi)=\sum_{n=1}^{N} I_{n} a_{m n} e^{j 2 \pi\left(f_{0}+m f_{n}\right) t} e^{j k\left(x_{n} u+y_{n} v\right)} .
$$

The power of main band $P_{0}$ and the total power of sideband $P_{S R}$ can be expressed as

$$
\begin{aligned}
P_{0} & =\int_{2 \pi}^{2 \pi} \int_{0}^{\pi}\left|E_{0}(\theta, \varphi)\right|^{2} \sin \theta \mathrm{d} \theta \mathrm{d} \varphi, \\
P_{S R} & =\sum_{m=-\infty, m \neq 0}^{m=\infty} \int_{0}^{2 \pi} \int_{0}^{\pi}\left|E_{m}(\theta, \varphi)\right|^{2} \sin \theta \mathrm{d} \theta \mathrm{d} \varphi,
\end{aligned}
$$

where $E_{0}(\theta, \varphi)$ is the electric field intensity of main band and $E_{m}(\theta, \varphi)$ is the electric field intensity of the $m$ th sideband.

The average power is the sum of the integral of power in all directions. According to equations (6) and (7), it can be defined as

$$
P_{a v}=\frac{P_{0}+P_{S R}}{4 \pi} .
$$

The directivity of time-modulated antenna array can be expressed as [22]

$$
D=\frac{4 \pi \max \left(\left|E_{0}(\theta, \varphi)\right|^{2}\right)}{P_{0}+P_{S R}} .
$$

2.2. Trapezoidal Pulse Time-Modulated Switching Function. Under the variable aperture sizes (VAS) modulation mode [23], the rectangular pulse switching function is usually used for time-modulated antenna array, as shown in Figure 2(a).

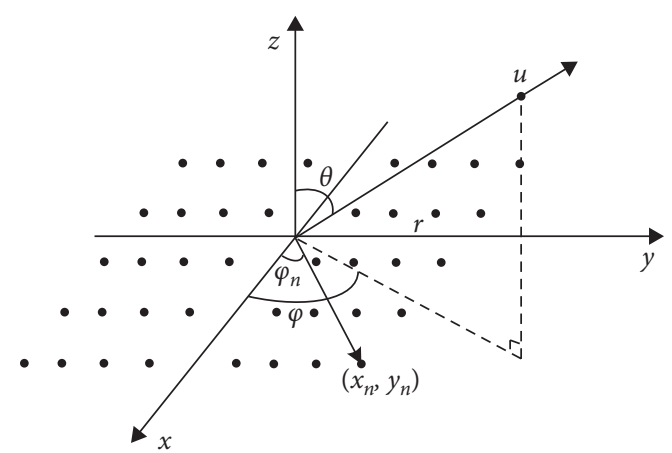

FIGURE 1: Planar arrays with square lattices and square boundaries.

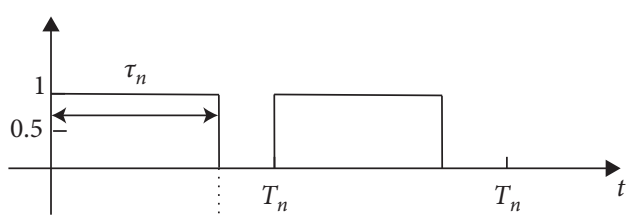

(a)

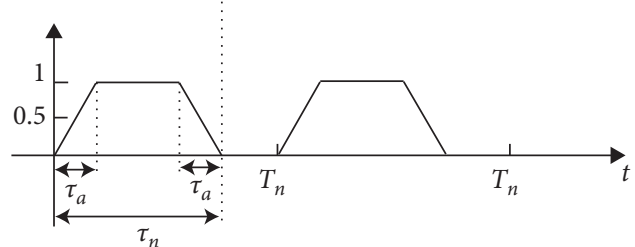

(b)

Figure 2: Time-pulse shaping. (a) Rectangular pulse. (b) Trapezoidal pulse.

As shown in Figure 2(b), the trapezoidal pulse switching function is obtained as follows:

$$
U_{n}(t)= \begin{cases}\frac{t}{\tau_{a},} & 0<t \leq \tau_{a}, \\ 1, & \tau_{a} \leq t \leq \tau_{n}-\tau_{a}, \\ \frac{\tau_{n}-t}{\tau_{a}}, & \tau_{n}-\tau_{a} \leq t \leq \tau_{n}, \\ 0, & \text { else. }\end{cases}
$$

The Fourier coefficient of the trapezoidal pulse switching function is (for details, see Appendix A)

$$
\begin{aligned}
a_{m n}= & \left(\tau_{n}-\tau_{a}\right) f_{n} \sin c\left(\pi m f_{n} \tau_{a}\right) \sin c\left[\pi m f_{n}\left(\tau_{n}-\tau_{a}\right)\right] \\
& \cdot e^{-j \pi m f_{n} \tau_{n}}, \quad 0<2 \tau_{a} \leq \tau_{n} \leq T_{n} .
\end{aligned}
$$

While the Fourier coefficient of the rectangular pulse switching function $U_{n}^{\prime}(t)=\left\{\begin{array}{ll}1, & 0 \leq t \leq \tau_{n} \\ 0, & \text { else }\end{array}\right.$ is

$$
a_{m n}^{\prime}=\tau_{n} f_{n} \sin c\left(\pi m f_{n} \tau_{n}\right) e^{-j \pi m f_{n} \tau_{n}}, \quad 0<\tau_{n} \leq T_{n} .
$$

Compared with rectangular pulse switching function, trapezoidal pulse switching function has better sideband 
suppression performance as its Fourier coefficient $a_{m n}$ has an extra $\sin c\left(\pi m f_{n} \tau_{a}\right)$ component.

A switch has only two state of ON and OFF, so in order to form trapezoidal pulse in reality, each element can be equipped with a radio frequency $(\mathrm{RF})$ variable gain amplifier (VGA). The periodic trapezoidal pulse of period $T_{n}$ governs the digitally controlled VGA instead of RF switches to transmit signal [24]. Compared with conventional timemodulated array based on RF switches, the power consumption is acceptable and the complexity of hardware structure is approximate [25]. Moreover, the VGAs guarantees flexibility and advanced dynamic control.

\subsection{The Mathematical Model of Shaped Beam Pattern} Synthesis. For side lobe and sideband suppression of sparse arrays based on time-modulated technology, the objective of this paper is to find an optimal solution for shaped beam pattern synthesis of sparse array. That is to optimize the array element position and excitation coefficient to make the pattern achieve the following goals: (a) minimize the peak side lobe level (PSLL) and peak sideband level (PSBL) under the constraint of main band; (b) minimize the number of elements; (c) make the dynamic range ratio (DRR) meet the constraint conditions. The abovementioned optimization problem can be expressed as

$$
\begin{array}{ll}
\min & \max _{\theta, \varphi \in \Omega_{s}}\left|E_{0}(\theta, \varphi)\right| \\
& \max \left|E_{m}(\theta, \varphi)\right|, m \neq 0 \\
& N \\
\text { s.t. } & \left|E_{0}(\theta, \varphi)-E_{d}(\theta, \varphi)\right|^{2}<\varepsilon_{1}, \theta, \varphi \in \Omega_{c} \\
& \min I_{n}=1, \max I_{n} \leq \varepsilon_{2},
\end{array}
$$

where $N$ is the number of array elements, $E_{0}(\theta, \varphi)$ is the electric field intensity of main lobe, $\Omega_{c}$ and $\Omega_{s}$ are the main lobe area and the side lobe area of main band, respectively, $E_{m}(\theta, \varphi)$ is the electric field intensity of sideband, $E_{d}(\theta, \varphi)$ is the desired shaped beam pattern of main band, $\varepsilon_{1}$ is the maximum relaxed coefficient of shaped beam pattern, and $\varepsilon_{2}$ is the maximum amplitude of the excitation coefficient.

\section{Hybrid Algorithm Based on Orthogonal Perturbation Method and Convex Optimization}

In order to meet the optimization objective of equation (13), this section decomposes equation (13) into four problems and uses different methods to optimize them, respectively: (a) under the constraints of the side lobe and sideband, the minimum number of elements is achieved by iteratively decreasing; (b) the suppression of sideband level is achieved by nonuniform periodic modulation and trapezoidal pulse switching function; the nonuniform periodic modulation can effectively suppress the PSBL, and the trapezoidal pulse switching function can further reduce the sideband level and sideband power radiation; (c) the array element position is optimized by the orthogonal perturbation method [26-28], and the optimized array realizes the sparse layout; (d) the equivalent excitation of array element is obtained by the convex optimization, and the joint optimization of equivalent excitation and position minimizes the PSLL. At the end of this section, the algorithms above are summarized and explained, and the general flowchart is given.

3.1. Minimum Number $N$ of Array Elements. According to the optimization objective of equation (13), the number of array elements needs to be minimized under the constraints of the side lobe and sideband. Under the condition that the array aperture remains invariant, the number of array elements is initialized to $N_{0}$, and the spacing of array elements meets $(\lambda / 2) . N_{0}$ can be written as

$$
N_{0}=\operatorname{ceil}\left(\frac{2 L_{x}}{\lambda}\right) \cdot \operatorname{ceil}\left(\frac{2 L_{y}}{\lambda}\right) \text {, }
$$

where $\left(L_{x}, L_{y}\right)$ is the aperture size of the planar array. In this paper, the minimum number of array elements is obtained by gradually reducing the array elements while the side lobe and sideband still meet the constraints.

3.2. Sideband Suppression Based on Trapezoidal Pulse Switching Function. According to the optimization objective of equation (13), the maximum value of the sideband electric field intensity $\left|E_{m}(\theta, \varphi)\right|, m \neq 0$ of the antenna array is required to be minimum. From equation (11), the amplitude of $a_{m n}$ decreases with the increase of $m$ in the function $\sin c(\cdot)$. That is, the sideband level decreases as the sideband order increases, so the sideband level of the array at $m=1$ is the highest [18]. This paper uses a nonuniform period timemodulated method [29], so the sideband energy of the array elements cannot be superimposed. Therefore, the PSBL of the array element is the PSBL of the array. Minimizing the PSBL is equivalent to

$$
\min \max _{n=1,2, \ldots N} a_{1 n}
$$

Under the condition that $\tau_{n}$ and $f_{n}$ are known, $a_{1 n}$ is only determined by $\tau_{a}$ in equation (11). According to the pattern synthesis algorithm of OPM-CVX proposed in Section 3.3, the best coefficients $I_{n} a_{0 n}$ can be obtained under the constraints of the main lobe and side lobe. That is, $I_{n} a_{0 n}=I_{n}\left(\tau_{n}-\tau_{a}\right) f_{n}$ is known when $m=0$. The value range of $\tau_{a}$ is $\left(0,\left(\tau_{n} / 2\right)\right)$. When $\tau_{a}=0$, the trapezoidal pulse switching function becomes the rectangular pulse switching function. The sideband suppression performance is the worst, while the radiated power of sidebands is the largest. When $\tau_{a}=\left(\tau_{n} / 2\right)$, the trapezoidal pulse switching function becomes a triangular pulse switching function, the sideband suppression performance is the best, while the radiated power of sidebands is the lowest. In this paper, the best rise/ fall time $\tau_{a}$ of trapezoidal pulse and the best static excitation $I_{n}$ are solved under the constraint of DRR $\min I_{n}=1, \max I_{n} \leq \varepsilon_{2}$ in equation (13) so that the radiated power of the main band and the suppression effect of the sideband are balanced. Under the constraints of $\min I_{n}=1, \max I_{n} \leq \varepsilon_{2}$, this paper initializes the $I_{n}$ of the array element firstly, and then design the value of $\tau_{a}$ under 
the balance requirement of main band radiated power and sideband suppression.

3.3. Orthogonal Perturbation Method for Optimal Location. According to the optimization objective of equation (13), the mean square error between the main lobe of the actual pattern and the main lobe of the desired pattern is required to be less than the set value, which can be expressed as

$$
\delta=\frac{1}{4 \pi} \int_{\Omega_{c}} \int_{\Omega_{c}}\left|E_{0}(\theta, \varphi)-E_{d}(\theta, \varphi)\right|^{2} \sin \theta \mathrm{d} \theta \mathrm{d} \varphi<\varepsilon_{1},
$$

where $\delta$ is the mean square error.

From equation (4), the electric field intensity of main band can be rewritten as

$$
E_{0}(\theta, \varphi)=\sum_{n=1}^{N} I_{n} a_{0 n} e^{j k\left(x_{n} u+y_{n} v\right)}=W^{T} B,
$$

where $B=\left[b_{1}, \ldots, b_{N}\right]^{T}, \quad b_{n}=e^{j k\left(x_{n} u+y_{n} v\right)}$ is the steering vector of the antenna array, $W=\left[w_{1}, \ldots, w_{N}\right]^{T}, w_{n}=I_{n} a_{0 n}$ is the excitation vector of the antenna array, and the carrier frequency common factor $e^{j 2 \pi f_{0} t}$ of main band is ignored.

Under the condition that the excitation vector $W$ is given, this paper uses orthogonal perturbation method to solve the optimal steering vector $B_{\text {best }}$, so as to obtain the optimal array layout. The idea is as follows: according to the total differential equation, when the excitations of array elements are known and the element position disturbance is small, the variation of equation (17) can be written as

$$
\begin{aligned}
\Delta E & =W^{T} \Delta B=\sum_{n=1}^{N} w_{n} \Delta B \\
& =\sum_{n=1}^{N} w_{n}\left(\Delta x_{n} \frac{\partial b_{n}}{\partial x_{n}}+\Delta y_{n} \frac{\partial b_{n}}{\partial y_{n}}\right) \\
& =\left(W_{e} \Delta S\right)^{T} B^{\prime},
\end{aligned}
$$

where $\Delta S=\left(\Delta x_{1}, \ldots, \Delta x_{N}, \Delta y_{1}, \ldots, \Delta y_{N}\right)$ is the perturbation of position information, $S=\left(x_{1}, \ldots, x_{N}, y_{1}, \ldots\right.$, $\left.y_{N}\right) . \quad B^{\prime}=\left(\left(\partial b_{1} / \partial x_{1}\right), \ldots,\left(\partial b_{N} / \partial x_{N}\right),\left(\partial b_{1} / \partial y_{1}\right), \ldots\right.$, $\left.\left(\partial b_{N} / \partial y_{N}\right)\right)$ is the direction of position perturbation, $W_{e}=\operatorname{diag}\left(w_{1}, \ldots, w_{N}, w_{1}, \ldots, w_{N}\right), Q$ is a set of standard orthogonal basis constructed by using Gram-Schmidt orthogonal process, which makes $B^{\prime}=C Q$. $C$ is the coefficient of orthogonal base, then equation (18) can be rewritten as

$$
\Delta E=\left(C^{T} W_{e} \Delta S\right)^{T} Q
$$

Assume $\Delta S_{c}=C^{T} W_{e} \Delta S$, after the element position disturbance, and equation (17) is converted to

$$
E_{0}(\theta, \varphi)=W^{T} B+\Delta E=W^{T} B+\left(\Delta S_{c}\right)^{T} Q
$$

Define the inner product of any two column vectors $x, y$ as

$$
\langle x, y\rangle=\frac{1}{4 \pi} \int_{0}^{2 \pi} \int_{0}^{\pi} x(\theta, \varphi) y^{H}(\theta, \varphi) \sin \theta \mathrm{d} \theta \mathrm{d} \varphi .
$$

Take equations (20) and (21) into (16), then

$$
\begin{aligned}
\delta= & \frac{1}{4 \pi} \int_{0}^{2 \pi} \int_{0}^{\pi}\left|W^{T} B-E_{d}(\theta, \varphi)+\left(\Delta S_{c}\right)^{T} Q\right|^{2} \sin \theta \mathrm{d} \theta \mathrm{d} \varphi \\
= & \left\langle W^{T} B-E_{d}(\theta, \varphi), W^{T} B-E_{d}(\theta, \varphi)\right\rangle \\
& +\left\langle W^{T} B-E_{d}(\theta, \varphi),\left(\Delta S_{c}\right)^{T} Q\right\rangle \\
& +\left\langle\left(\Delta S_{c}\right)^{T} Q, W^{T} B-E_{d}(\theta, \varphi)\right\rangle+\left\langle\left(\Delta S_{c}\right)^{T} Q,\left(\Delta S_{c}\right)^{T} Q\right\rangle .
\end{aligned}
$$

According to the method for calculating the derivative of scalar function to complex vector [30], the partial derivative to $\Delta S_{c}$ is zero, that is,

$$
\frac{\partial \delta}{\partial \Delta S_{c}}=\langle Q, \Delta E\rangle+\Delta S_{c}=0
$$

The iterative process is introduced by the difference $\Delta E_{i}$ so that the actual pattern can constantly approach the desired pattern. According to $\Delta S=W_{e}^{-1} C^{-T} \Delta S_{c}$, on the $i+1$ iteration, the position is updated to

$$
S_{i+1}=S_{i}+\Delta S_{i+1}=S_{i}-W_{e}^{-1} C_{i}^{-T}\left\langle Q_{i}, \Delta E_{i}\right\rangle
$$

Therefore, when the excitation vector $W$ is determined, the optimal element position $S_{\text {best }}$ can be solved by the orthogonal perturbation method.

3.4. Convex Optimization for Optimal Excitation Vector. According to the optimization objective of (13), the maximum value of the side lobe electric field intensity $\left|E_{0}(\theta, \varphi)\right|, \theta, \varphi \in \Omega_{s}$ of the antenna array is required to be minimum. For a given array layout, that is, when the steering vector of antenna array is known, the problem of solving $\min \left(\theta, \varphi \in \Omega_{s}{ }^{\max }\left|W^{T} B\right|\right)$ is a convex problem [31]. The best excitation vector $W_{\text {best }}$ can be solved with an available tool, such as CVX [4].

3.5. The Procedure of Proposed Algorithm. For time-modulated sparse planar array, the step by step implementation method is proposed to achieve the objectives mentioned in Section 3.1-3.4. The four objectives are minimizing the number of array elements, achieving sideband suppression and radiant power balance design, realizing shaped beam of main lobe, and suppressing side lobe level, respectively. This paper is to find the optimal array layout and excitation and modulated timing sequence to meet the abovementioned four objectives. Figure 3 is the flowchart of the proposed algorithm.

Figure 3 shows the implementation process of the minimum number of array elements, sideband suppression and radiant power balance design, shaped beam of main lobe, and side lobe level constraint. The optimization process of the array starts with the initialization of the shaped beam pattern, the PSLL and PSBL, the initialization of the number $N$, position $\left(x_{n}, y_{n}\right)$ and the excitation amplitude $I_{n}$ of the array element, the initialization of the switch-on duration time $\tau_{n}$, the rise/fall time $\tau_{a}$, and the modulation 


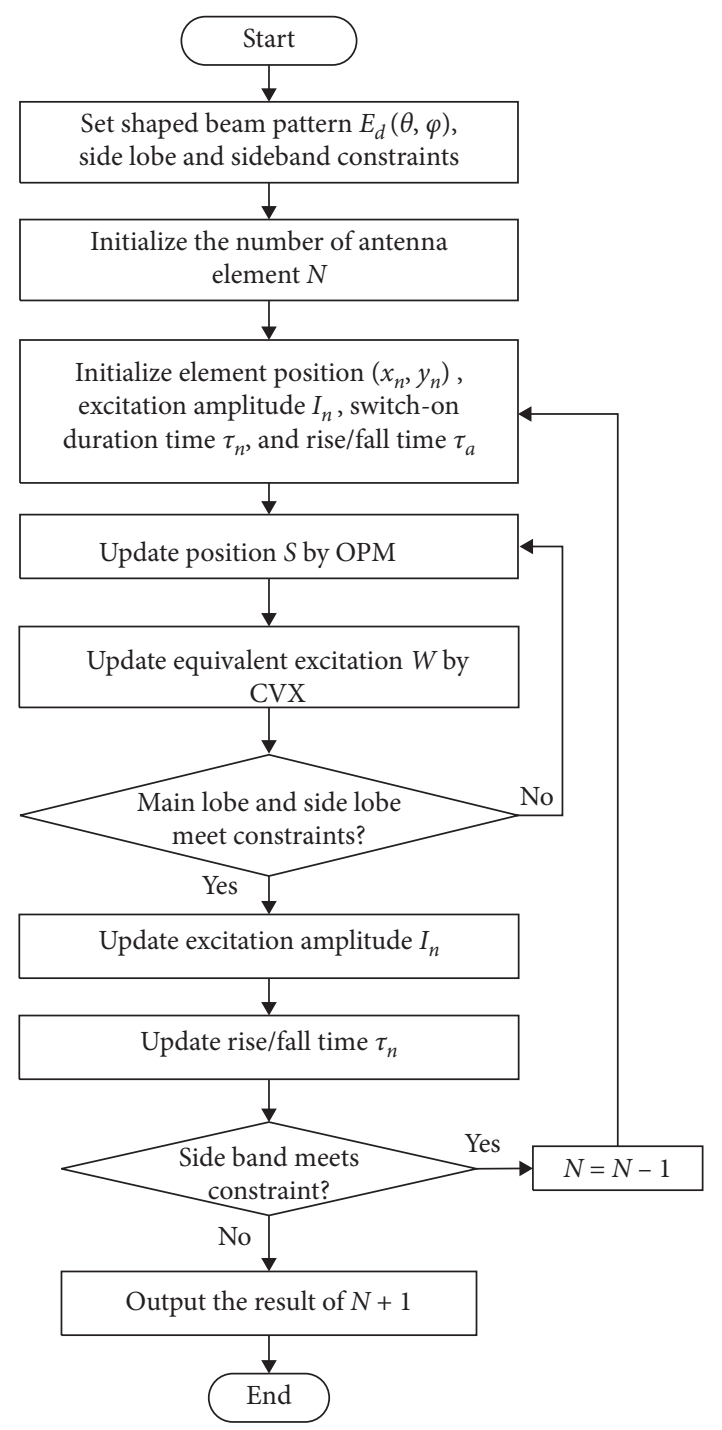

FIGURE 3: Flowchart of the proposed algorithm.

frequency $f_{n}$. The initial array layout is distributed at equal intervals of $(\lambda / 2)$. The array elements are gradually reduced to find the minimum number of array elements that meets the sideband level constraint. The solving steps of the algorithm are (1) update the excitation amplitude according to the element's equivalent excitation obtained by the optimization of the main band; (2) update the rise/fall time $\tau_{a}$; (3) calculate the PSBL of the array using the nonuniform period modulation method; (4) reduce the number of array elements iteratively and make $N=N-1$. When the sideband constraint cannot be satisfied, output the result of $N=N+1$.

In Step (1), the OPM-CVX algorithm is used to iteratively update the position and equivalent excitation of the array element. The specific process is (a) construct orthogonal basis by Gram-Schmidt orthogonal process; (b) update the perturbation location parameters; (c) adopt convex algorithm to optimize the excitation coefficient and reduce PSLL with the position information; (d) calculate the actual electric field intensity according to the updated position and excitation; (e) loop iteratively until the actual electric field intensity meets the constraints of the shaped beam pattern and side lobe.

\section{Simulation Results}

In this section, a variety of simulations and comparisons are presented in order to illustrate the advantages of the proposed hybrid algorithm. The simulations in this paper are achieved by a PC with CPU model i7-6500U, main frequency $2.5 \mathrm{GHz}$, and RAM of $8.00 \mathrm{~GB}$.

4.1. Side Lobe and Sideband Suppression. Under the constraint that the main lobe pattern is consistent, two examples are presented in comparison with the results of pencil beam and flat top beam pattern obtained in $[12,15]$ to demonstrate the effectiveness of the proposed algorithm in suppressing the PSBL and PSLL. In the first example, A linear array of 9element with initial equal spacing of $0.5 \lambda$ is studied. In the case that the main lobe pattern is consistent with the one in [15], the objective is to minimize the PSLL and PSBL. Similar simulations have been previously carried out by the efficient mathematical method (EMM) in [15]. The optimized PSLL and PSBL were $-22 \mathrm{~dB}$ and $-15.2 \mathrm{~dB}$, respectively. With the $0.2^{\circ}$ sampling interval of $\theta$, the optimization process takes 2 iterations and 0.784 seconds. Under the condition that number of array elements and the array aperture are the same, the PSLL and PSBL optimized by proposed algorithm are $-22.42 \mathrm{~dB}$ and $-15.6 \mathrm{~dB}$, respectively, which are lower than the values in [15]. The main band and the first sideband patterns, the histogram of the excitation amplitude of array elements, are shown in Figures 4 and 5, respectively. Table 1 shows the specific value of values of position and excitation. Because the positions and excitations of the elements are centrosymmetric, only 5 sets of data are listed in Table 1. Table 2 shows the comparison of the parameters optimized by the proposed algorithm and the EMM in [15].

The second example studies a linear array of 30 elements with initial equal spacing of $0.6 \lambda$. The flat top beam pattern with a main lobe width of $60^{\circ}$ is set as the desired pattern. The objective is to minimize the PSLL and PSBL. Patra et al. [12] used DE algorithm to synthesize the same array and obtained the PSLL of $-26.46 \mathrm{~dB}$ and the PSBL of $-40.49 \mathrm{~dB}$, respectively. In this paper, the PSBL of $-26.66 \mathrm{~dB}$ and the PSLL of $-47.51 \mathrm{~dB}$ are obtained by OPM-CVX algorithm. The optimized ripple of flat top beam is $0.965 \mathrm{~dB}$, slightly higher than $0.7224 \mathrm{~dB}$ in [12]. Figure 6 shows the synthesized pattern by OPM-CVX and DE. Figure 7 shows the amplitude and position of array elements by OPM-CVX.

With the $0.2^{\circ}$ sampling interval of $\theta$, the optimization process takes 2 iterations and 0.806 seconds. The spacing of the two elements closest to the centre of the array is $0.2004 \lambda$, and the spacing of other elements is greater than $0.5 \lambda$. The minimum equivalent excitation is 0.011 . The detailed comparison of parameters is shown in Table 3. By comparison, the proposed algorithm obtains a smaller aperture size and a better flat top beam under the equal amplitude in-phase excitation.

The comparison results with the pattern in $[12,15]$ shows that the OPM-CVX algorithm with nonuniform period 


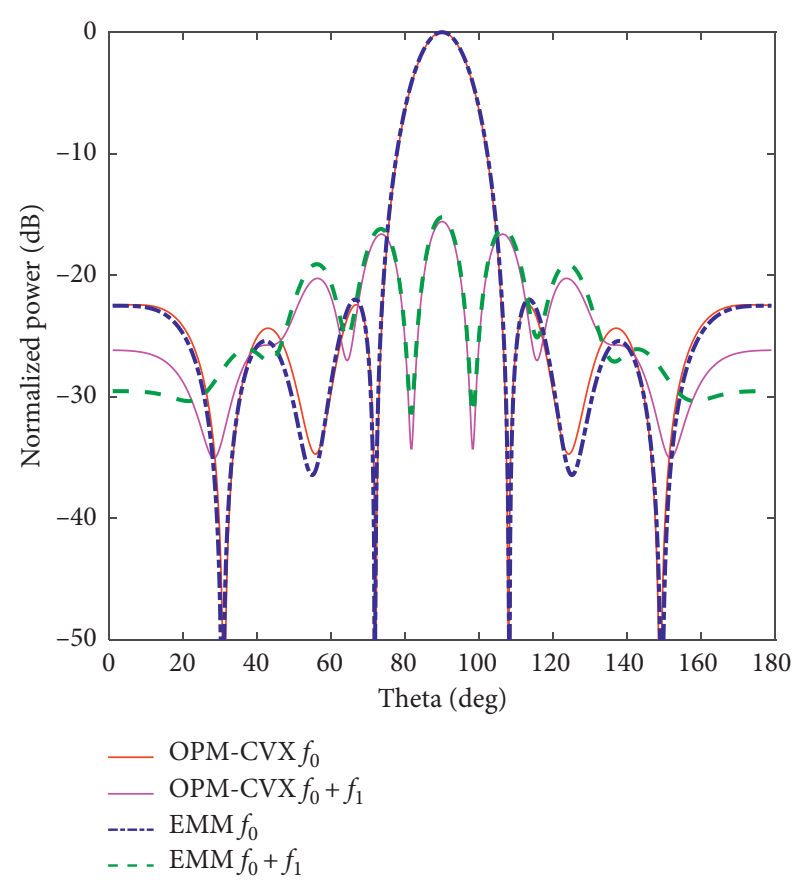

Figure 4: Comparison between the designed array and the one in [15].

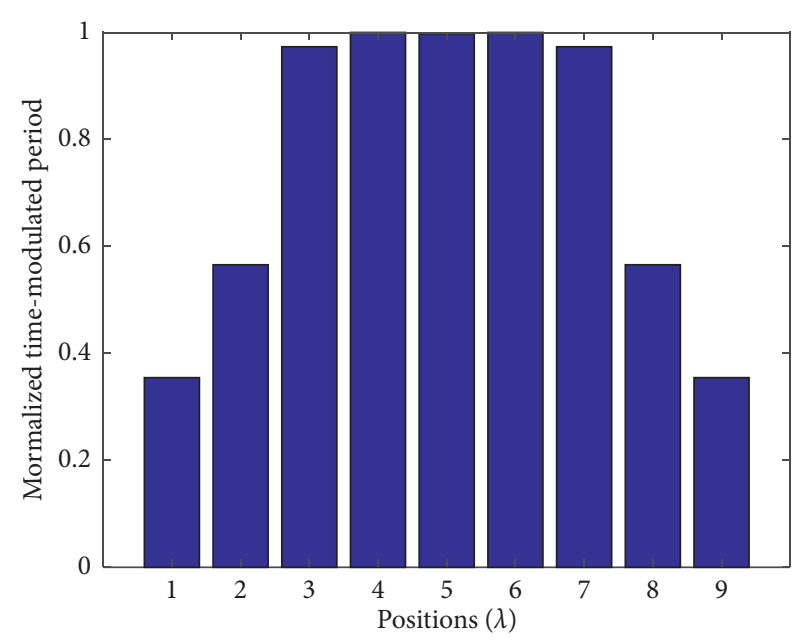

FIGURE 5: Excitation amplitude optimized by OPM-CVX.

TABle 1: Position and excitation of array elements.

\begin{tabular}{lccccc}
\hline Element No. & 5 & 6 & 7 & 8 & 9 \\
\hline Position $[\lambda]$ & 0 & 0.4829 & 0.9688 & 1.4724 & 2.0000 \\
Excitation & 0.9976 & 1 & 0.9733 & 0.5656 & 0.3546 \\
\hline
\end{tabular}

time-modulated mode can obtain better effect of pattern synthesis.

4.2. Minimization of the Number of Array Elements. In order to demonstrate the effectiveness of the OPM-CVX algorithm in reducing the number of the array elements, two examples are presented in comparison with the results in $[18,21,32,33]$. The first example studies a planar array of 100
$(10 \times 10)$ elements with equal spacing of $0.5 \lambda$. The optimization objective is to reduce the element number and suppress the PSBL under the side lobe constraint of $-20 \mathrm{~dB}$. Similar simulations have been previously carried out in [21,32], but only the array elements with an equal spacing of $0.5 \lambda$ were considered. Under the aperture constraint of $5 \lambda \times 5 \lambda$, the number of array elements synthesized by the proposed algorithm is $64(8 \times 8)$. The PSBL is $-42.27 \mathrm{~dB}$, lower than $-31.8 \mathrm{~dB}$ in [21] and $-34.69 \mathrm{~dB}$ in [32]. Figure 8 shows the main band pattern. Figure 9 shows the first sideband pattern, and it is essentially the first sideband pattern of a certain array element. Because the array element is isotropic, the electric field intensity is consistent in the $\theta$ and $\varphi$ directions.

With the $1^{\circ}$ and $2^{\circ}$ sampling interval of $\theta$ and $\varphi$, The optimization process takes 30 iterations and 449.8 seconds. Compared with the linear array above, the planar array increases the number of array elements and the complexity of the array, so the time consumption and the number of iterations in the optimization process are increased. The comparison of parameters is shown in Table 4. The element positions are shown in Figure 10 and listed in Table 5. The minimum element spacing of $x$ direction and $y$ direction is $0.6877 \lambda$, and the maximum element spacing of $x$ direction and $y$ direction is $0.7089 \lambda$. The optimized aperture of $8 \times 8$ planar array is $4.9007 \lambda \times 4.8986 \lambda$.

The second example studies a planar array with 400 $(20 \times 20)$ elements. The elements are arranged with square lattices and square boundaries. The optimization objective is to minimize the number of elements under the side lobe constraint of $-42 \mathrm{~dB}$. Similar simulations have been previously carried out in $[18,33]$. The comparison of two-dimensional pattern between the proposed algorithm and the PCS-CVX in [18] is shown in Figure 11. The optimized PSLL is $-43.17 \mathrm{~dB}$, and the optimized element number is 324 , which is $10 \%$ less than that in [18].

With the $1^{\circ}$ and $2^{\circ}$ sampling interval of $\theta$ and $\varphi$, The optimization process takes 12 iterations and 1981.8 seconds. Compared with the $8 \times 8$ planar array, the increase of array elements further increases the computational complexity and time consumption. The minimum element spacing of $x$ direction and $y$ direction is $0.8446 \lambda$, and the maximum element spacing of $x$ direction and $y$ direction is $0.8507 \lambda$. The optimized aperture of $18 \times 18$ planar array is $14.4499 \lambda \times 14.4501 \lambda$. The element positions are shown in Figure 12. The detailed comparison of parameters is shown in Table 6 .

Through the experiments compared with [18, 21, 32, 33], the nonuniform period time-modulated mode and OPMCVX algorithm can effectively reduce the element number and suppress the PSBL, with the constraints of the main lobe response and the side lobe level.

4.3. Analysis of the Rise/Fall Time, Sideband, and Power. Two examples are presented to verify the effect of the rise/fall time of the trapezoidal pulse switching function on the sideband performance and to achieve the power balance between sideband and main band under the constraint of DRR. The first example shows the relationship between the sideband level and the rise/fall time. The same 9-element 
TABLe 2: Performance comparison between the designed array and the actual one in [15].

\begin{tabular}{lccccc}
\hline Method & Number of Elements & PSLL $(\mathrm{dB})$ & PSBL $(\mathrm{dB})$ & Aperture Size $[\lambda]$ & Time consumed $(\mathrm{s})$ \\
\hline EMM in [15] & 9 & -22 & -15.2 & 4 & - \\
OPM-CVX & 9 & -22.42 & -15.6 & 4 & 0.784 \\
\hline
\end{tabular}

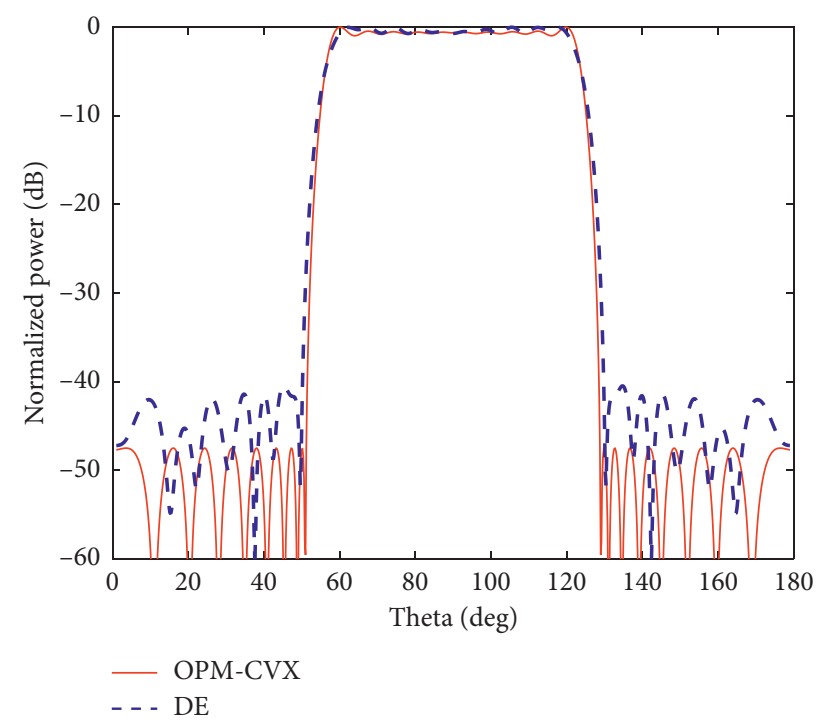

FIgURE 6: Comparison between the designed array and the one in [12].

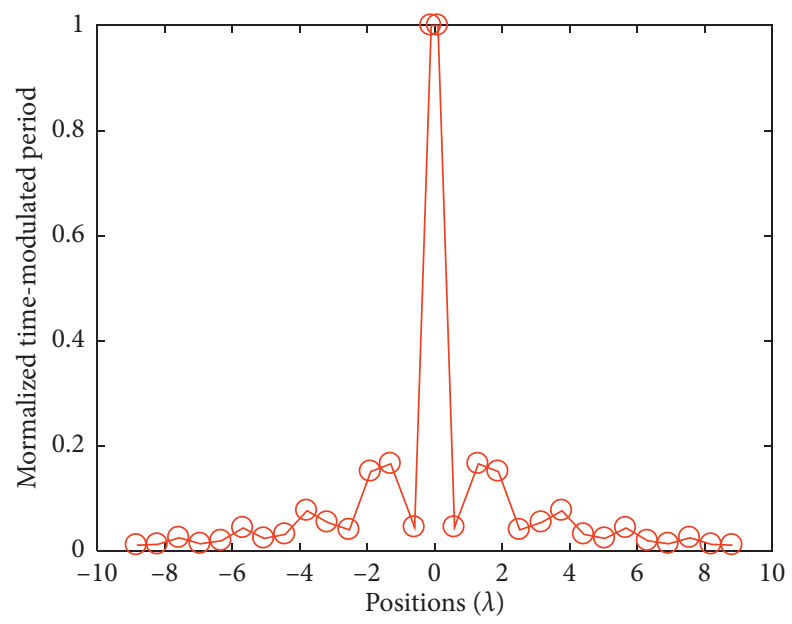

FIgURE 7: Excitation amplitude and position optimized by OPM-CVX.

TABLe 3: Performance comparison between the designed array and the actual one in [12].

\begin{tabular}{lcccccc}
\hline Algorithm & Number of Elements & PSLL (dB) & PSBL (dB) & MAX Ripple (dB) & DRR & Aperture $[\lambda]$ \\
\hline DE in [12] & 30 & -40.4932 & -26.4655 & 0.7224 & 6.2289 \\
OPM-CVX & 30 & -47.51 & -26.66 & 0.965 & 19.5904 \\
\hline
\end{tabular}

linear array as that in Section 4.1 is adopted. From the synthesis results of the 9-element linear array, the normalized minimum switch-on duration time is $\tau_{n}=0.3546$. According to the value range $\tau_{a} \in\left(0,\left(\tau_{n} / 2\right)\right)$, we can get $\tau_{a} \leq 0.1773$. Figure 13 shows the first 20 sideband curves corresponding to different $\tau_{a}$. The sideband level in this figure decreases as $\tau_{a}$ increases. Table 7 shows the relationship between the power of the main band and the sideband level when $\tau_{a}$ is different. As $\tau_{a}$ increases, the sideband level decreases faster. The power of main band is obtained by equation (6).

As shown in Figure 14, the higher harmonic reduces faster when applying trapezoidal pulse and the power reduction is higher as $\tau_{a}$ increase, until the electric field 


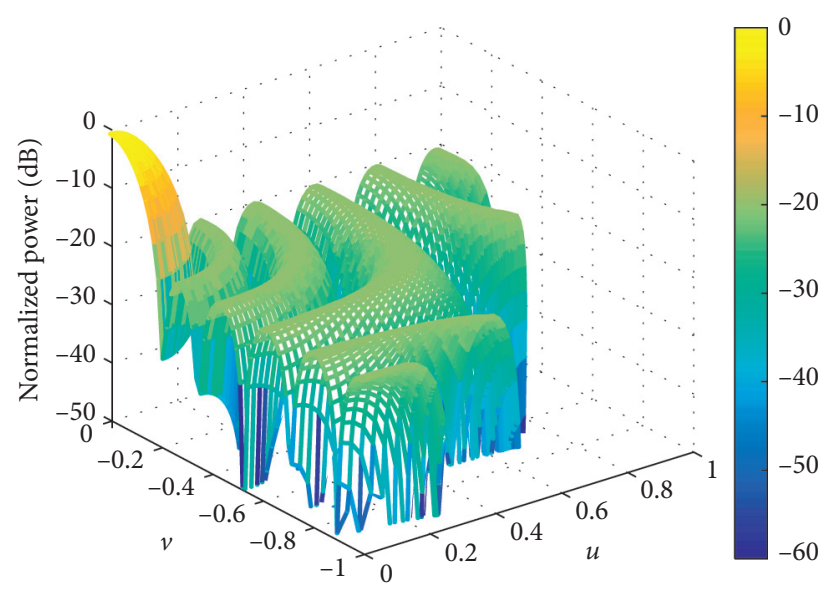

FIGURE 8: Designed array of $8 \times 8$.

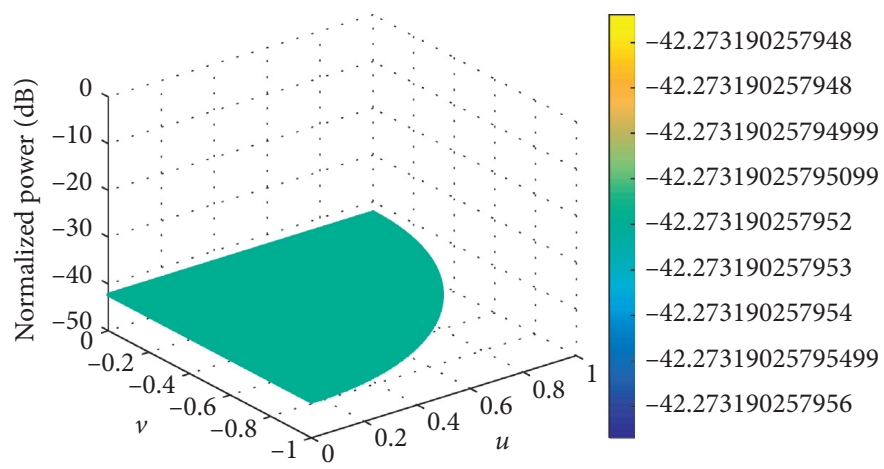

Figure 9: First sideband of $8 \times 8$.

TABle 4: Performance comparison between the designed array and the actual one in $[21,32]$.

\begin{tabular}{lccccc}
\hline Algorithm & PSLL $(\mathrm{dB})$ & PSBL $(\mathrm{dB})$ & Number of elements & Position $[\lambda]$ & Aperture $[\lambda]$ \\
\hline PSO in [21] & -19.6 & -31.8 & 76 & 0.5 & $5 \times 5$ \\
DE in [32] & -19.86 & -34.69 & 80 & 0.5 & $5 \times 5$ \\
OPM-CVX & -19.90 & -42.27 & 64 & Table 5 & $4.9007 \times 4.8986$ \\
\hline
\end{tabular}

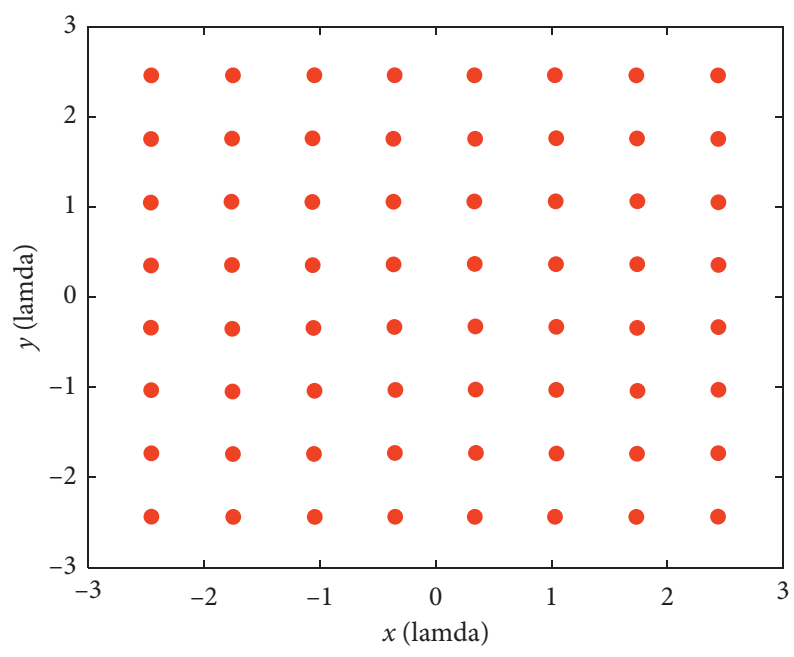

FIGURE 10: Element position of $8 \times 8$ planar array. 
TABLe 5: Optimized element position.

\begin{tabular}{ccccccccc}
\hline & $y_{1}[\lambda]$ & $y_{2}[\lambda]$ & $y_{3}[\lambda]$ & $y_{4}[\lambda]$ & $y_{5}[\lambda]$ & $y_{6}[\lambda]$ & $y_{7}[\lambda]$ & $y_{8}[\lambda]$ \\
\hline$x_{1}[\lambda]$ & $(-2.45-2.45)$ & $(-2.45-1.75)$ & $(-2.45-1.04)$ & $(-2.45-0.35)$ & $(-2.450 .34)$ & $(-2.451 .04)$ & $(-2.451 .74)$ & $(-2.452 .45)$ \\
$x_{2}[\lambda]$ & $(-1.74-2.45)$ & $(-1.74-1.75)$ & $(-1.75-1.06)$ & $(-1.75-0.36)$ & $(-1.750 .34)$ & $(-1.751 .05)$ & $(-1.751 .75)$ & $(-1.742 .45)$ \\
$x_{3}[\lambda]$ & $(-1.04-2.45)$ & $(-1.04-1.75)$ & $(-1.04-1.05)$ & $(-1.05-0.35)$ & $(-1.050 .34)$ & $(-1.061 .05)$ & $(-1.051 .75)$ & $(-1.042 .45)$ \\
$x_{4}[\lambda]$ & $(-0.34-2.45)$ & $(-0.34-1.74)$ & $(-0.34-1.04)$ & $(-0.35-0.34)$ & $(-0.360 .35)$ & $(-0.361 .05)$ & $(-0.361 .74)$ & $(-0.352 .45)$ \\
$x_{5}[\lambda]$ & $(0.35-2.45)$ & $(0.36-1.74)$ & $(0.35-1.04)$ & $(0.35-0.34)$ & $(0.350 .36)$ & $(0.341 .05)$ & $(0.351 .74)$ & $(0.342 .45)$ \\
$x_{6}[\lambda]$ & $(1.04-2.45)$ & $(1.05-1.75)$ & $(1.05-1.04)$ & $(1.05-0.34)$ & $(1.050 .35)$ & $(1.041 .05)$ & $(1.051 .75)$ & $(1.042 .45)$ \\
$x_{7}[\lambda]$ & $(1.74-2.45)$ & $(1.75-1.75)$ & $(1.75-1.05)$ & $(1.75-0.35)$ & $(1.750 .34)$ & $(1.751 .05)$ & $(1.751 .75)$ & $(1.742 .45)$ \\
$x_{8}[\lambda]$ & $(2.45-2.45)$ & $(2.45-1.74)$ & $(2.45-1.04)$ & $(2.45-0.34)$ & $(2.450 .35)$ & $(2.451 .04)$ & $(2.451 .74)$ & $(2.452 .45)$ \\
\hline
\end{tabular}

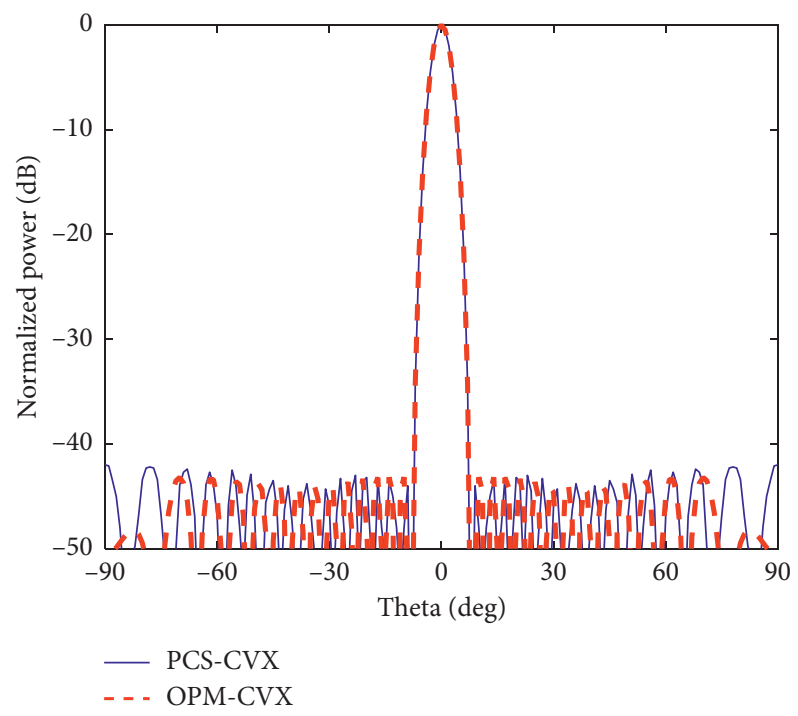

Figure 11: Comparison of 2D normalized pattern with the one in [18].

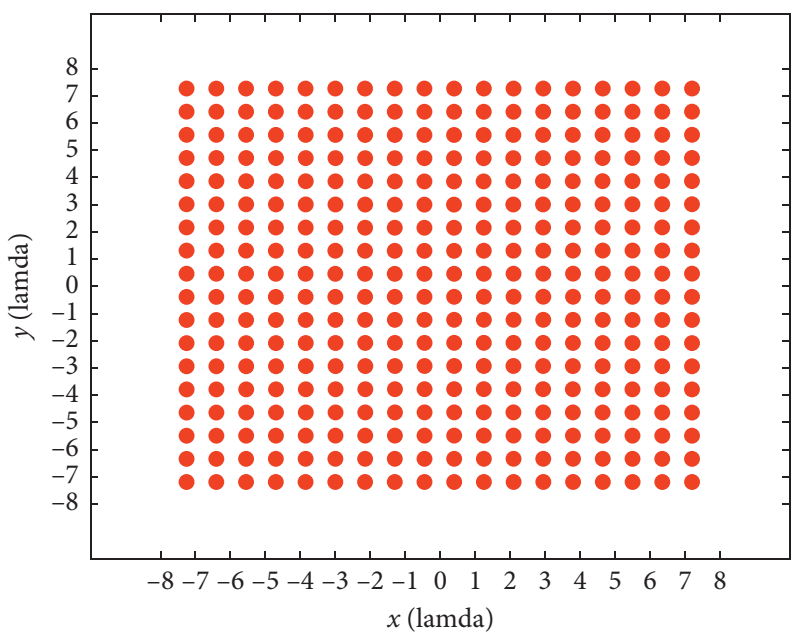

Figure 12: Element position of $18 \times 18$ planar array.

TABle 6: Performance comparison between the designed array and the actual one in $[18,33]$.

\begin{tabular}{lcccccc}
\hline Algorithm & Gain $(\mathrm{dB})$ & Number of elements & PSLL $(\mathrm{dB})$ & PSBL $(\mathrm{dB})$ & $d_{\min }[\lambda]$ & Aperture $[\lambda]$ \\
\hline$[18]$ & 29.63 & 358 & -42 & - & 0.6295 & $15.6 \times 15.6$ \\
{$[33]$} & - & 496 & -41 & - & $<0.5$ & $10 \times 10$ \\
OPM-CVX & 32.00 & 324 & -43.17 & -49.39 & 0.847 & $14.4499 \times 14.4501$ \\
\hline
\end{tabular}




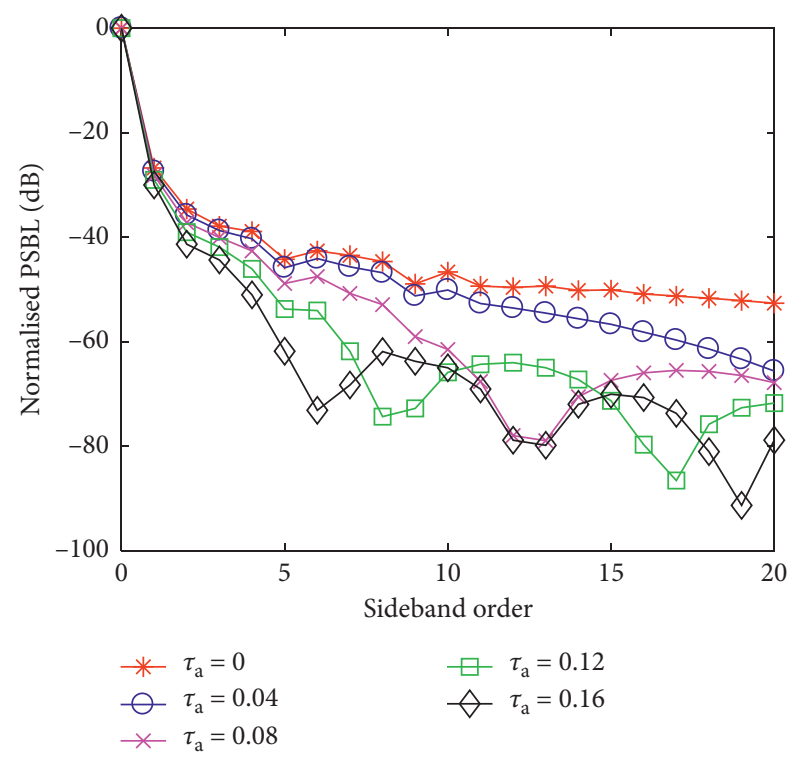

Figure 13: Comparison of the first 20 order sidebands under different $\tau_{a}$.

TABLE 7: Relationship between $\tau_{a}$, sideband level, and power of main band.

\begin{tabular}{lccccccc}
\hline$\tau_{a}$ & Power of main band $(\mathrm{dB})$ & 1st SBL $(\mathrm{dB})$ & 2nd SBL $(\mathrm{dB})$ & 3rd SBL $(\mathrm{dB})$ & 4th SBL $(\mathrm{dB})$ & 5th SBL $(\mathrm{dB})$ & 6th SBL $(\mathrm{dB})$ \\
\hline 0 & 70.5526 & -26.7594 & -34.6214 & -37.8957 & -38.9044 & -44.2325 \\
0.04 & 69.7273 & -26.7823 & -34.7130 & -38.1024 & -39.2733 & -44.8117 & -42.6294 \\
0.08 & 68.8667 & -27.4881 & -36.0300 & -39.3719 & -41.4599 & -47.6923 & -46.8514 \\
0.12 & 67.9685 & -28.2907 & -37.6815 & -41.1580 & -44.7104 & -52.3954 & -53.3080 \\
0.16 & 67.0302 & -29.1995 & -39.7258 & -43.5518 & -49.4283 & -60.4416 & -72.3277 \\
\hline
\end{tabular}

intensity goes to zero. Table 8 shows the power of main band, the power of the first 8 sidebands and the directivity of 9element linear array, which are calculated by equations (6), (7), and (9), respectively. Comparing $\tau_{a}=0.16$ with $\tau_{a}=0$, the reduction of the first 8 sideband power turns out to be around $21.73 \%$. Meanwhile, the decrease of directivity is very small. Therefore, the increase of $\tau_{a}$ reduces the power of the main band but improves the radiation efficiency. According to reference [22], since the directivity is almost constant in Table 8 , the gain of antenna array is improved.

In the second example, the same $64(8 \times 8)$ elements planar array as that in Section 4.2 is adopted. From the synthesis results of the $64(8 \times 8)$ elements planar array, the normalized minimum switch-on duration time is $\tau_{n}=0.03$. Under the condition that the power of the main band is constant, changing the DRR from 1 to 8 , then the rise/fall time $\tau_{a}$ of the trapezoidal switching function can be set to a maximum value of 0.12 . Figure 15 shows the first 20 sidebands optimized by the rectangular switching function, trapezoidal switching function when $\tau_{a}=0.12$. It can be seen that the sideband suppression effect of the trapezoidal pulse switching function is better than the rectangular pulse switching function and the DE algorithm in [32].

As shown in Figure 16 and Table 9, the power variation trend of the main band and sidebands of the planar array are the same as that of the linear array. Comparing $\tau_{a}=0.12$ with $\tau_{a}=0$, the reduction in the first 8 sideband power turns out to be around 5.35\%. According to reference [22], because of the

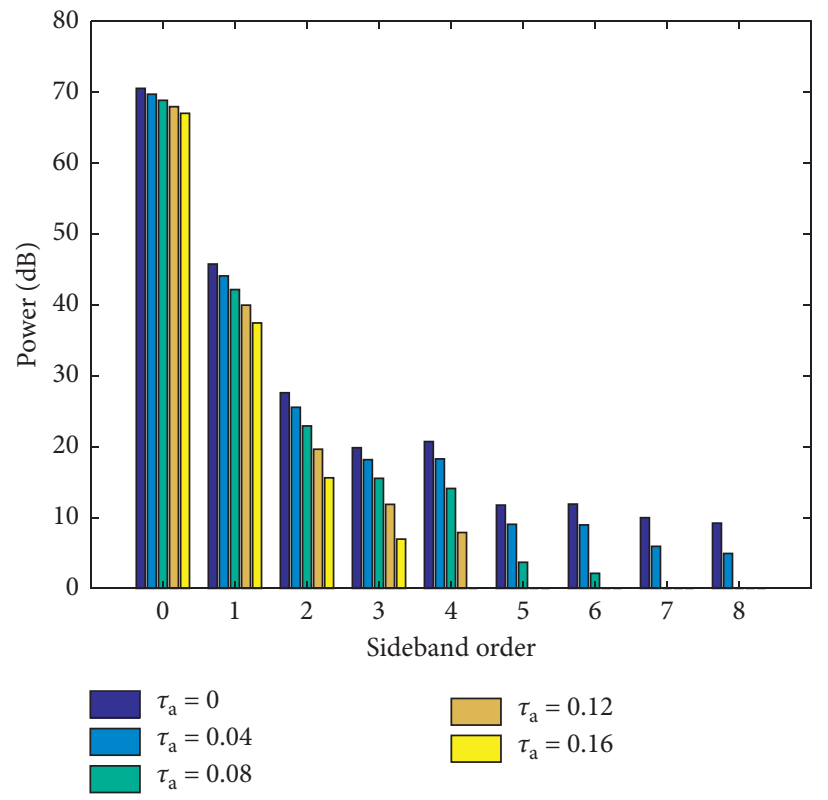

FIgURE 14: Power comparison of the main band and the first 8 order sidebands under different $\tau_{a}$.

tiny decrease of directivity and the large increase of main band radiation efficiency, the gain of antenna array is improved.

According to the simulations of linear array and planar array, the trapezoidal pulse switching function has better 
TABLe 8: Power of the first 8 sidebands and main band under different $\tau_{a}$.

\begin{tabular}{|c|c|c|c|c|c|}
\hline$\tau_{a}$ & 0 & 0.04 & 0.08 & 0.12 & 0.16 \\
\hline Power of main band $(\mathrm{dB})$ & 70.55 & 69.73 & 68.87 & 67.97 & 67.03 \\
\hline Power of first 8 sidebands $(\mathrm{dB})$ & 156.74 & 134.92 & 100.51 & 79.33 & 60.00 \\
\hline Radiation efficiency of main band (\%) & 31.04 & 34.07 & 40.66 & 46.14 & 52.77 \\
\hline Directivity & 27.19 & 27.16 & 27.13 & 27.10 & 27.04 \\
\hline
\end{tabular}

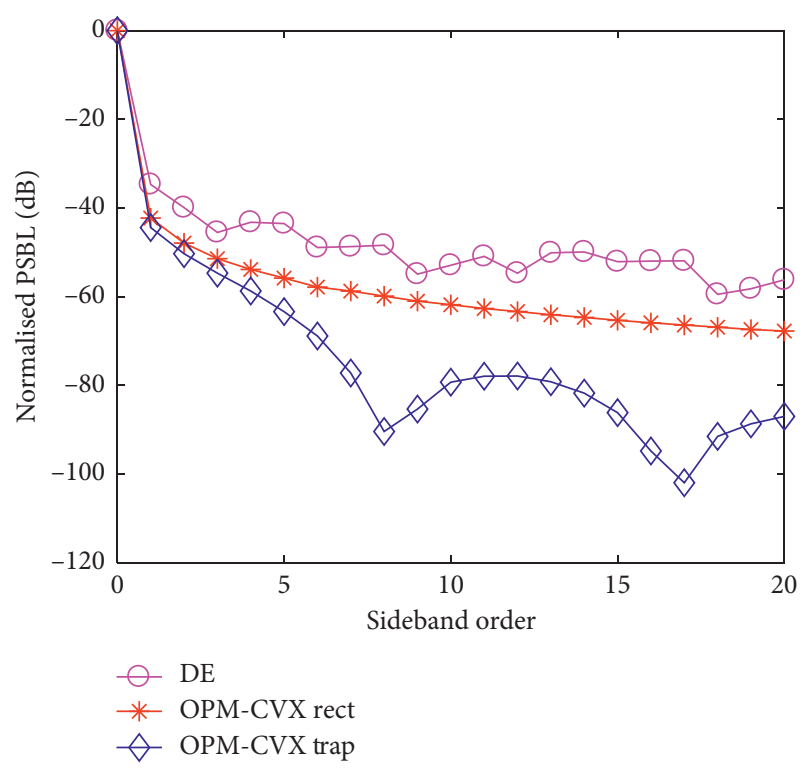

Figure 15: Comparison of the first 20 order sidebands optimized by OPM-CVX and DE.

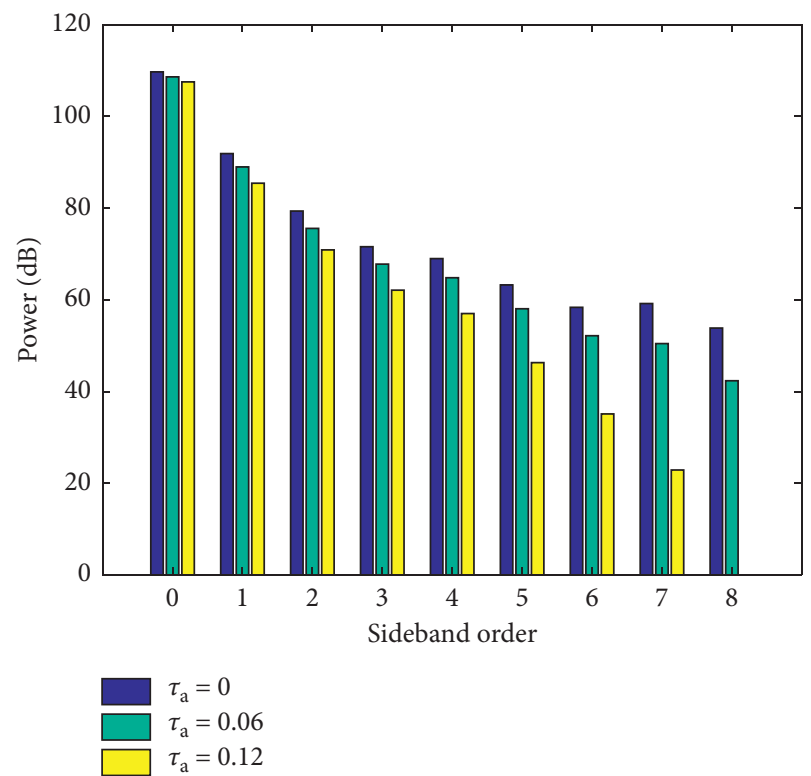

Figure 16: Power comparison of the main band and the first 8 sidebands under different $\tau_{a}$.

sideband suppression effect than the rectangular pulse switching function. The sideband power decreases faster than the main band power as $\tau_{a}$ increases, and the radiation efficiency of the main band is improved. Therefore, the increase of $\tau_{a}$ can effectively suppress the sideband radiation
TABLE 9: Power of the first 8 sideband and main band under different $\tau_{a}$.

\begin{tabular}{lccc}
\hline$\tau_{a}$ & 0 & 0.06 & 0.12 \\
\hline Power of main band $(\mathrm{dB})$ & 109.71 & 108.62 & 107.53 \\
Power of first 8 sidebands (dB) & 546.50 & 500.22 & 379.79 \\
Radiation efficiency of main band (\%) & 16.72 & 17.84 & 22.07 \\
Directivity & 22.42 & 22.27 & 22.07 \\
\hline
\end{tabular}

and improve the radiation efficiency and gain of the main band. In addition, the power loss of the main band caused by the increase of $\tau_{a}$ can be reduced by increasing the DRR.

\section{Conclusion}

In time-modulated antenna array, the high side lobe will lower the radiation performance of the main lobe, and the redundant sideband will reduce the radiation efficiency of the main band. In order to solve these problems, a hybrid OPM-CVX algorithm is proposed to suppress the side lobe of sparse array, and the nonuniform periodic time-modulated mode and trapezoidal pulse switching function are used to suppress sideband. In addition, under the constraint of the shaped beam of main lobe, the minimum array elements, low side lobe, and sideband are realized step by step in the way of loop iteration strategy. The simulation results of linear and planar arrays show that the proposed algorithm can achieve shaped beam of main lobe and low side lobe with fewer array elements. The algorithm can achieve the balanced design of sideband suppression and the main band power by adjusting the DRR of the antenna array and the rise/fall time of the trapezoidal pulse switching function. Compared with other commonly used algorithms, the proposed algorithm can improve the performance of the pattern of time-modulated sparse array and have important research value and practicability.

\section{Appendix}

\section{The derivation process of the Fourier coefficient $a_{m n}$}

The Fourier coefficient $a_{m n}$ of the trapezoidal pulse switching function can be derived by differentiating the switching function. The periodic piecewise-linear waveforms have the following characteristics [34]. (1) Any waveform or function can be written as a linear combination of two or more functions, so the trapezoidal periodic function can be disassembled into a simple linear combination of square wave function and saw-tooth wave function. (2) The Fourier coefficient of the impulse function is the reciprocal of the 


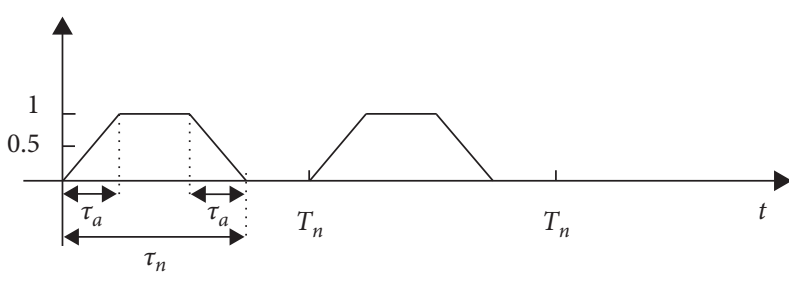

(a)

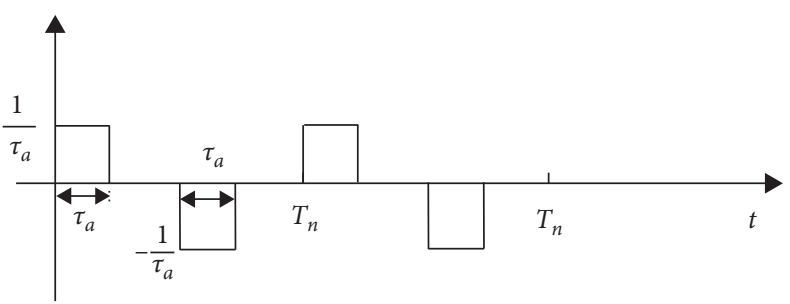

(b)

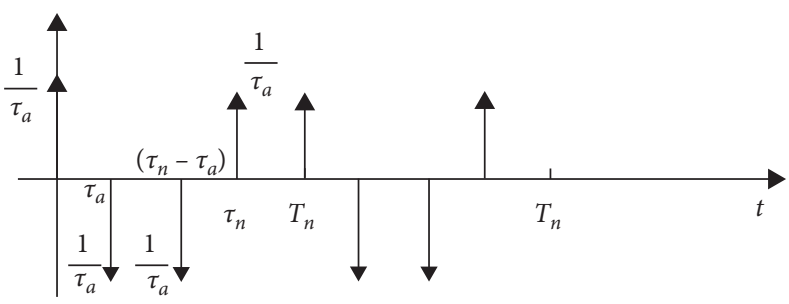

(c)

FIgURe 17: Derivative of trapezoidal pulse: (a) trapezoidal pulse, (b) first derivative and (c) second derivative.

period. (3) There is a transformational relation between the Fourier coefficient of periodic switching function $U_{n}(t)$ and the Fourier coefficients of its various derivatives. The $k$ th derivative of equation 3 is represented as

$$
\frac{\mathrm{d}^{k} U_{n}(t)}{\mathrm{d} t^{k}}=\sum_{m=-\infty}^{+\infty} a_{m n}^{(k)} e^{j 2 \pi m f_{p} t},
$$

where $a_{m n}=\left(1 /\left(j 2 \pi m f_{p}\right)^{k}\right) a_{m n}^{(k)}, a_{m n}^{(k)}$ is the Fourier coefficient of the $n$th element's $k$ thderivative of the $m$ th harmonic. According to the above three properties, the trapezoidal pulse switch function is differentiated repeatedly until the first impulse function appears. If the differential function contains more than just one impulse function, the parts that are not impulse functions can be differentiated continuously until all terms are impulse functions.

From the derivative relation of square wave function, saw-tooth wave function, and impulse function, the square wave function in Figure 17(b) is the derivative of the sawtooth wave function shown in Figure 17(a), and the impulse function in Figure 17(c) is the derivative of the square wave function shown in Figure 17(b). There is only the impulse function in Figure 17(c), so the Fourier coefficient of the second derivative of the trapezoidal wave is

$$
\begin{aligned}
a_{m n}^{(2)}= & \frac{1}{T} \frac{1}{\tau_{a}}-\frac{1}{T} \frac{1}{\tau_{a}} e^{-j 2 \pi m f_{n} \tau_{a}}-\frac{1}{T} \frac{1}{\tau_{a}} e^{-j 2 \pi m f_{n}\left(\tau_{n}-\tau_{a}\right)}+\frac{1}{T} \frac{1}{\tau_{a}} e^{-j 2 \pi m f_{n} \tau_{n}} \\
= & \frac{1}{T}\left(\begin{array}{c}
\frac{1}{\tau_{a}} e^{-j \pi m f_{n} \tau_{a}}\left(e^{j \pi m f_{n} \tau_{a}}-e^{-j \pi m f_{n} \tau_{a}}\right) \\
-\frac{1}{\tau_{a}} e^{-j \pi m f_{n} \tau_{a}} e^{-j 2 \pi m f_{n}\left(\tau_{n}-\tau_{a}\right)}\left(e^{j \pi m f_{n} \tau_{a}}-e^{-j \pi m f_{n} \tau_{a}}\right)
\end{array}\right) \\
= & j \frac{1}{2 \pi m}\left(m \omega_{0}\right)^{2} \frac{\sin \pi m f_{n} \tau_{a}}{\pi m f_{n} \tau_{a}} e^{-j \pi m f_{n} \tau_{n}} \\
& \cdot\left(e^{j \pi m f_{n}\left(\tau_{n}-\tau_{a}\right)}-e^{-j \pi m f_{n}\left(\tau_{n}-\tau_{a}\right)}\right) .
\end{aligned}
$$

Then, the Fourier coefficient of the trapezoidal pulse switching function is

$$
\begin{aligned}
a_{m n} & =\frac{1}{\left(j m \omega_{0}\right)^{2}} a_{m n}^{(2)} \\
& =-j \frac{1}{2 \pi m} e^{-j \pi m f_{n} \tau_{n}} \frac{\sin \pi m f_{n} \tau_{a}}{\pi m f_{n} \tau_{a}}\left(e^{j \pi m f_{n}\left(\tau_{n}-\tau_{a}\right)}-e^{-j \pi m f_{n}\left(\tau_{n}-\tau_{a}\right)}\right) \\
& =\left(\tau_{n}-\tau_{a}\right) f_{n} \frac{\sin \pi m f_{n} \tau_{a}}{\pi m f_{n} \tau_{a}} \frac{\sin \pi m f_{n}\left(\tau_{n}-\tau_{a}\right)}{\pi m f_{n}\left(\tau_{n}-\tau_{a}\right)} e^{-j \pi m f_{n} \tau_{n}} \\
& =\left(\tau_{n}-\tau_{a}\right) f_{n} \sin c\left(\pi m f_{p} \tau_{a}\right) \sin c\left(\pi m f_{p}\left(\tau_{n}-\tau_{a}\right)\right) e^{-j \pi m f_{n} \tau_{n}} .
\end{aligned}
$$

In order to keep the power constant, $\tau_{a} \leq \tau_{n}$ needs to be satisfied when trapezoidal pulse switching function is used instead of rectangular pulse switching function. The DRR of the array can be adjusted appropriately to increase $\tau_{n}$ when $\tau_{n}$ is small so that $\tau_{a}$ can reach a wider value range.

\section{Data Availability}

The data used to support the findings of this study are available from the corresponding author upon request.

\section{Conflicts of Interest}

The authors declared no potential conflicts of interest with respect to the research, authorship, and/or publication of this article.

\section{Acknowledgments}

This work was supported by the National Natural Science Foundation of China (Grant no. 61671239) and Fundamental Research Funds for the Central Universities (Grant no. kfjj20190419). 


\section{References}

[1] B. Pyne, P. R. Akbar, V. Ravindra, F. Saito, J. Hirokawa, and T. Fukami, "Slot-array antenna feeder network for spaceborne," IEEE Transactions on Antennas and Propagation, vol. 66, no. 7, pp. 3463-3474, 2018.

[2] H. Kikuchi, T. Ushio, F. Mizutani, and M. Wada, "Improving the accuracy of rain rate estimates using X-band phased-array weather radar network," IEEE Transactions on Geoscience and Remote Sensing, vol. 56, no. 12, pp. 6986-6994, 2018.

[3] H. A. Diawuo and Y. Jung, "Broadband proximity coupled microstrip planar antenna array for 5G cellular applications," Antennas and Wireless Propagation Letters, vol. 17, no. 7, pp. 1286-1290, 2019.

[4] S. Lei, H. Hu, B. Chen, P. Tang, J. Tian, and X. Qiu, "An array position refinement algorithm for pencil beam pattern synthesis with high-order taylor expansion," IEEE Antennas and Wireless Propagation Letters, vol. 18, no. 9, pp. 1766-1770, 2019.

[5] P. Cao, J. S. Thompson, and H. Haas, "Constant modulus shaped beam synthesis via convex relaxation," IEEE Antennas and Wireless Propagation Letters, vol. 16, pp. 617-620, 2016.

[6] Y.-X. Qi and J.-Y. Li, "Superposition synthesis method for 2-D shaped-beam array antenna," IEEE Transactions on Antennas and Propagation, vol. 66, no. 12, pp. 6950-6957, 2018.

[7] A. F. Morabito, "Synthesis of maximally-sparse square or rectangular arrays through compressive sensing," in Proceedings of the 2017 11th European Conference on Antennas and Propagation (EUCAP), pp. 2829-2832, Paris, France, 2017.

[8] H. Shen, B. H. Wang, and X. Li, "Shaped-beam pattern synthesis of sparse linear arrays using the unitary matrix pencil method," IEEE Antennas and Wireless Propagation Letters, vol. 16, pp. 1098-1101, 2016.

[9] F. Yan, F. Yang, P. Yang, and T. Dong, "Synthesis of planar sparse arrays by perturbed compressive sampling framework," IET Microwaves, Antennas \& Propagation, vol. 10, no. 11, pp. 1146-1153, 2016.

[10] L. Poli, P. Rocca, A. Massa, and M. D’Urso, “Optimized Design of Sparse Time Modulated Linear Arrays," in Proceedings of the 2013 7th European Conference on Antennas and Propagation (EuCAP), pp. 138-141, Gothenburg, Sweden, 2013.

[11] A. Reyna, L. I. Balderas, and M. A. Panduro, "Time-modulated antenna arrays for circularly polarized shaped beam patterns," IEEE Antennas and Wireless Propagation Letters, vol. 16, no. 12, pp. 1537-1540, 2017.

[12] S. Patra, S. K. Mandal, G. K. Mahanti, and N. Pathak, " "Synthesis of flat-top power pattern in time-modulated unequally spaced linear arrays using DE," in Proceeidngs of the 2015 IEEE 2nd International Conference on Recent Trends in Information Systems (ReTIS), pp. 104-108, Kolkata, India, 2015.

[13] M. D’Urso, A. Iacono, and A. Iodice, “Optimizing uniformly equally spaced and sparse excited time-modulated linear arrays," in Proceedings of the 2011 IEEE Radar Conference (RADAR), pp. 586-589, Kansas City, MO, USA, 2011.

[14] L. Poli, P. Rocca, L. Manica, and A. Massa, "Pattern synthesis in time-modulated linear arrays through pulse shifting," IET Microwaves, Antennas \& Propagation, vol. 4, no. 9, pp. 1157-1164, 2010.

[15] O. Gassab, A. Azrar, A. Dahimene, and S. Bouguerra, "Efficient mathematical method to suppress sidelobes and sidebands in time-modulated linear arrays," IEEE Antennas and Wireless Propagation Letters, vol. 18, no. 5, pp. 836-840, 2019.
[16] L. F. Yepes, D. H. Covarrubias, M. A. Alonso, and R. Ferrus, "Hybrid sparse linear array synthesis applied to phased antenna arrays," IEEE Antennas and Wireless Propagation Letters, vol. 13, pp. 185-188, 2014.

[17] J. I. Echeveste, M. A. Gonzalez de Aza, and J. Zapata, "Shaped beam synthesis of real antenna arrays via finite-element method, floquet modal analysis, and convex programming," IEEE Transactions on Antennas and Propagation, vol. 64, no. 4, pp. 1279-1286, 2016.

[18] C. Yan, P. Yang, Z. Xing, and S. Y. Huang, "Synthesis of planar sparse arrays with minimum spacing constraint," IEEE Antennas and Wireless Propagation Letters, vol. 17, no. 6, pp. 1095-1098, 2018.

[19] J. Rubio, J. Corcoles, J. F. Izquierdo, and R. Gomez-Alcala, "Array thinning of coupled antennas based on the orthogonal matching pursuit method and a spherical-wave expansion for far-field synthesis," IEEE Transactions on Antennas and Propagation, vol. 63, no. 12, pp. 5425-5432, 2015.

[20] J. Y. Li, Y. X. Qi, and S. G. Zhou, "Shaped beam synthesis based on superposition principle and taylor method," IEEE Transactions on Antennas and Propagation, vol. 65, no. 17, pp. 6157-6160, 2017.

[21] L. Poli, P. Rocca, L. Manica, and A. Massa, "Time modulated planar arrays - analysis and optimisation of the sideband radiations," IET Microwaves, Antennas \& Propagation, vol. 4, no. 9, pp. 1165-1171, 2010.

[22] S. Yang, Y. B. Gan, and P. K. Tan, "Evaluation of directivity and gain for time-modulated linear antenna arrays," Microwave and Optical Technology Letters, vol. 42, no. 2, pp. 167171, 2004.

[23] W. Kummer, A. Villeneuve, T. Fong, and F. Terrio, "Ultralow sidelobes from time-modulated arrays," IEEE Transactions on Antennas and Propagation, vol. 11, no. 6, pp. 633639, 1963.

[24] L. Poli, P. Rocca, G. Oliveri et al., "Advanced pulse sequence design in time-modulated arrays for cognitive radio," IEEE Antennas and Wireless Propagation Letters, vol. 17, no. 5, pp. 898-902, 2018.

[25] R. Maneiro-Catoira, J. Bregains, J. Garcia-Naya et al., "Enhanced time-modulated arrays for harmonic beamforming," IEEE Journal of Selected Topics in Signal Processing, vol. 11, no. 2, pp. 259-270, 2017.

[26] A. I. Uzkov, "An approach to the problem of optimum directive antenna design," Doklady an USSR, vol. 53, no. 1, pp. 35-38, 1946.

[27] H. Unz, "Nonuniformly spaced arrays: the orthogonal method," Proceedings of the IEEE, vol. 54, no. 1, pp. 53-54, 1966.

[28] J. Sahalos, "A solution of the general nonuniformly spaced antenna array," Proceedings of the IEEE, vol. 62, no. 9, pp. 1292-1294, 1974.

[29] I. Kanbaz, U. Yesilyurt, and E. Aksoy, "A study on harmonic power calculation for nonuniform period linear time modulated arrays," IEEE Antennas and Wireless Propagation Letters, vol. 17, no. 12, pp. 2369-2373, 2018.

[30] J. E. Gentle, Matrix Algebra: Theory, Computations, and Applications in Statistics, Springer, Gainesville, FL, USA, 1nd edition, 2007.

[31] S. Boyd and L. Vandenberghe, Convex Optimization, Cambridge, New York, NY, USA, 2nd edition, 2004.

[32] E. Aksoy and E. Afacan, "An inequality for the calculation of relative maximum sideband level in time-modulated linear and planar arrays," IEEE Transactions on Antennas and Propagation, vol. 62, no. 6, pp. 3392-3397, 2014. 
[33] W. Zhang, L. Li, and F. Li, "Reducing the number of elements in linear and planar antenna arrays with sparseness constrained optimization," IEEE Transactions on Antennas and Propagation, vol. 59, no. 8, pp. 3106-3111, 2011.

[34] C. R. Paul, Introduction to Electromagnetic Compatibility, Wiley, Hoboken, NJ, USA, 2nd edition, 2007. 